\title{
Three-Dimensional Multiple-Order Twinning of Self-Catalyzed GaAs Nanowires on Si Substrates
}

\author{
Emanuele Uccelli, ${ }^{+,}$Jordi Arbiol, ${ }^{\ddagger}, \boldsymbol{\oplus}$ Cesar Magen, ${ }^{\S}$ Peter Krogstrup," Eleonora Russo-Averchi, ${ }^{\dagger}$
} Martin Heiss, ${ }^{\dagger}$ Gabriel Mugny, ${ }^{\dagger}$ François Morier-Genoud, ${ }^{\perp}$ Jesper Nygård,,$"$ Joan Ramon Morante, ${ }^{\#, \nabla}$ and Anna Fontcuberta i Morral ${ }^{*,+}$

\author{
${ }^{\dagger}$ Laboratoire des Matériaux Semiconducteurs, Ecole Polytechnique Fédérale de Lausanne, 1015 Lausanne, Switzerland \\ ${ }^{\ddagger}$ Institució Catalana de Recerca i Estudis Avançats (ICREA) and Institut de Ciència de Materials de Barcelona, ICMAB-CSIC, \\ E-08193 Bellaterra, Catalonia, Spain \\ §Instituto de Nanociencia de Aragon-ARAID and Departamento de Física de la Materia Condensada, Universidad de Zaragoza, \\ 50018 Zaragoza, Spain \\ "Nano-Science Center, Niels Bohr Institute, University of Copenhagen, 2100 Copenhagen, Denmark \\ ${ }^{\perp}$ Laboratoire d'Optoelectronique Quantique, Ecole Polytechnique Federale de Lausanne, 1015 Lausanne, Switzerland \\ \#Catalonia Institute for Energy Research, IREC, 08930 Sant Adrià del Besòs, Spain \\ $\nabla$ Department d'Electrònica, Universitat de Barcelona, 08028 Barcelona, Spain
}

Supporting Information

ABSTRACT: In this paper we introduce a new paradigm for nanowire growth that explains the unwanted appearance of parasitic nonvertical nanowires. With a crystal structure polarization analysis of the initial stages of GaAs nanowire growth on $\mathrm{Si}$ substrates, we demonstrate that secondary seeds form due to a three-dimensional twinning phenomenon. We derive the geometrical rules that underlie the multiple growth directions observed experimentally. These rules help optimizing nanowire array devices such as solar or water splitting cells or of more complex hierarchical branched nanowire devices.
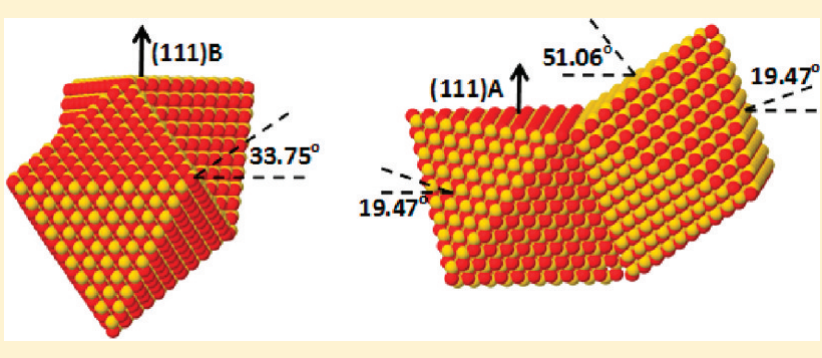

KEYWORDS: Nanowires, three-dimensional twinning, nanowire growth mechanisms, III-V on silicon, epitaxy, polarity

$\mathrm{T}$ he integration of III-V nanowires (NWs) on silicon and 1 germanium has excited a whole area of research and technology as it enables the integration of highly functional materials on the CMOS platform. ${ }^{1-7}$ Although the self-organized growth of III-V NWs on silicon has been intensively studied in the last years, ${ }^{8-12}$ important issues that concern the polarity mismatch between III-V NWs and the group IV substrate remain unsolved. The pioneering works of Atwater and Lewis show that nanowire arrays are at the forefront of solving the renewable energy challenge of the 21 st century. ${ }^{13,14}$ Unfortunately, defects such as nonvertical NWs occur often and result in the deterioration of the performance of nanowire array devices such as solar cells since the nonvertical nanowires provide undesired leakage paths. ${ }^{15}$ Only with a fundamental understanding of the elementary processes occurring at the initial stage of nanowire growth it will become possible to obtain a completely successful integration of III-V nanowires on group IV substrates. It has been predicted $^{16}$ and recently demonstrated ${ }^{17,18}$ that NW growth is 2D nucleation limited at the liquid-solid interface ${ }^{6}$ and that the nucleation takes place preferentially at the triple phase line. Consequently, the growth dynamics are highly dependent on changes in the morphology of the liquid phase, ${ }^{19}$ which determines not only whether the structure becomes wurtzite or zinc blende ${ }^{20}$ but also why kinking and crawling phenomena occur. ${ }^{21-23}$ Twinning has also been revealed as an important issue, as it can lead to crystalline phase change and kinking. ${ }^{24-26}$ The consequences for the electronic transport of the existence of twinning and a mixture in crystal phase have been demonstrated. $^{27}$ The existence of crystallographically equivalent twin boundaries with opposite polar bonding across the interface would lead to even stronger perturbations of electronic structure of the material. ${ }^{28}$ However, the issue of polarity conservation across the twin has only been raised occasionally, mainly due to the difficulties in its determination. ${ }^{29}$

Orthodox theories assume that the initial stage of vaporliquid-solid (VLS) growth of nanowires takes place at the liquid-solid interface forming a monocrystalline seed. ${ }^{17}$ This phenomenon is consistent with the growth of nanowires in epitaxial relation with the substrate. However, nanowires grown in other directions always appear, especially when $\mathrm{III}-\mathrm{V}$ materials

Received: June 5, 2011

Revised: August 4, 2011

Published: August 08, 2011 
a)

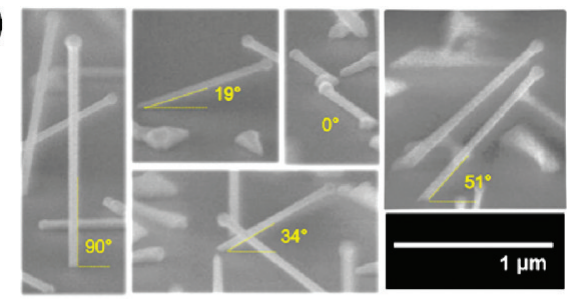

b)

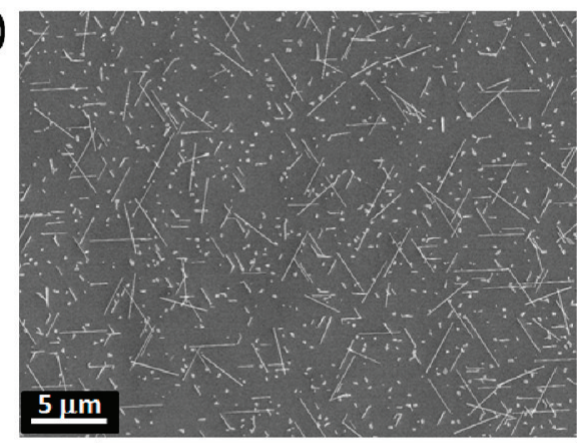

c)

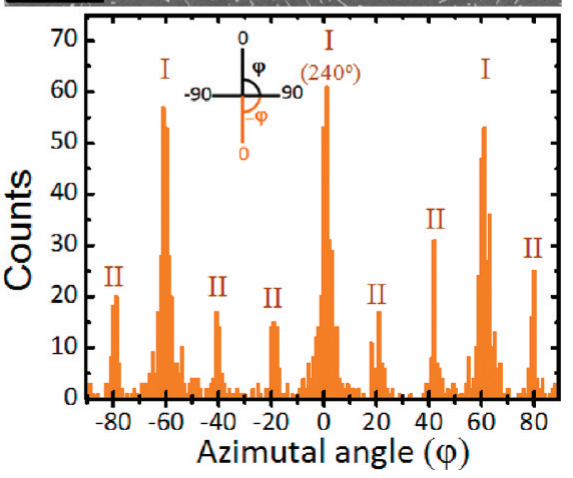

Figure 1. (a) Cross-section scanning electron micrographs of GaAs nanowires growing in different angles with respect to the substrate surface. Nanowires formed with $90^{\circ}$ angle, results from the growth from a single crystalline seed with B polarity (As terminated), while the $19^{\circ}$ growth direction results from the growth from a single crystalline seed with A polarity (Ga terminated). The rest of growth directions can be explained with multiple order twinning: the $0^{\circ}$ angle is a result of multiple $3 \mathrm{D}$ twinning on a seed with $\mathrm{B}$ polarity, while 34 and $51^{\circ}$ originate from a second order twinning with seeds exhibiting $B$ and $A$ polarity, respectively. (b) Planar view of a field of nanowires grown under V/III ratio of 30 . The multiple orientations of the nonvertical wires are clear. (c) Histogram of the nonvertical nanowire orientations found from the analysis of 500 nanowires grown under the same conditions as in (a) and (b). The occurrence of different directions is quantized, in agreement with our $3 \mathrm{D}$ twinning theory.

are grown on group IV materials such as silicon or germanium. ${ }^{30,31}$ Following the orthodox theory, for self-catalyzed nanowires growing always in the $[111] \mathrm{B}$ direction, only two types of growth orientations should be observed when grown on group IV (111) substrates. For a single seed with B polarity, i.e., an As terminated (111) surface, the nanowires should always grow vertically at $90^{\circ}$ with respect to the substrate surface. For a single seed with A polarity, i.e., a Ga terminated (111) surface, nanowires should turn into a growth direction of $19.47^{\circ}$ from the substrate (see S1 in Supporting Information). Our results shown here question the single crystalline nature of the seed assumed so far and impose a paradigm shift of nanowire growth. Here we present an explanation and experimental evidence of a manifold of growth directions, which are typically found to occur and which

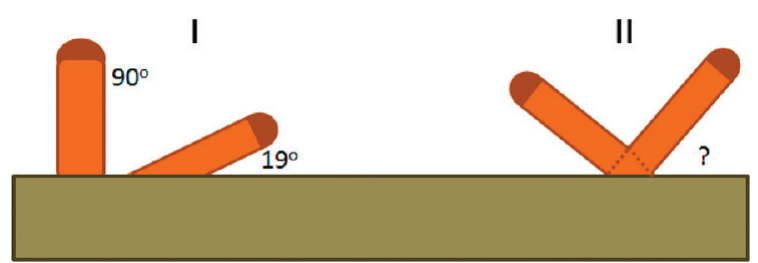

Figure 2. Schematics of the two types of growth obtained. Type I refers to the cases where a direct crystallographic relation between the nanowire growth direction and the substrate is found and type II when three-dimensional twinning is found. We draw the case in which two nanowires grow from the same point (like in Figure.3), but this does not have to be the case for type II.

are not in direct epitaxial relation with the substrate (cf. Figure 1). Using aberration corrected high angle annular dark field (HAADF) scanning transmission electron microscopy, we observe the polarity of the nanowire structures and the occurrence of $3 \mathrm{D}$ twinning at the initial stage of GaAs nanowire growth provoking nonepitaxial growth relations with the Si substrate. The results are general to the III-V semiconductor family and could potentially be extended to other substrates than silicon.

GaAs nanowires were grown on a 2 in. $\mathrm{Si}(111)$ undoped substrate by the gallium-assisted method ${ }^{32,33}$ using a DCA P600 MBE machine. The wafers were transferred directly from the wafer box to the MBE machine without any removal of the native oxide. Prior to growth, the substrates were degassed at $770{ }^{\circ} \mathrm{C}$ for $30 \mathrm{~min}$. The nanowires were obtained under rotation of $7 \mathrm{rpm}$ at a temperature of $620^{\circ} \mathrm{C}$ under a flux of Ga equivalent to a planar growth rate of $0.3 \AA / \mathrm{s}$. The $\mathrm{V} / \mathrm{III}$ ratio was varied between 15 and 60. The Ga and As fluxes were opened at the same time. We used reflection high-energy electron diffraction (RHEED) in situ for the detection of the nanowire nucleation, which corresponds to the onset after which electron diffraction signal from the nanowires is obtained. This corresponds to a size of the nucleation seeds of $10-20 \mathrm{~nm}$. The morphology of the samples was characterized by scanning electron microscopy (SEM). After cross sections were prepared with a focused ion beam, the structure was investigated by aberration corrected high angle annular dark field (HAADF) scanning transmission electron microscopy (STEM) imaging. 3D atomic models have been obtained by using the Rhodius software package. $^{34}$

Figure 1a contains representative cross-section scanning electron microscopy (SEM) images of nanowires grown on $\mathrm{Si}(111)$ under a V/III ratio of 30 . Under these conditions we find that $53 \%$ of the nanowires grow vertically and about $6 \%$ with a $19^{\circ}$ angle growing away from the substrate and $6 \%$ with a $19^{\circ}$ angle growing toward the substrate (the growth then often stops leading to the formation of a triangular object or it continues further following the substrate surface). Interestingly, we find that $41 \%$ of the nanowires grow in other directions that are quantized. The growth angles, which are different from 90, 19, or $-19^{\circ}$ (e.g., $34^{\circ}$ or $51^{\circ}$ ), are inconsistent with the crystallography of a single seed. The occurrence of quantized families of angles can be observed in a more precise way in a planar view SEM image of a nanowire field, such as the one shown in Figure $1 \mathrm{~b}$. Typically, one would expect the nonvertical wires would form families of angles separated by $120^{\circ}$. Clearly, in Figure $1 \mathrm{~b}$ one observes many more of them. In Figure 1c, we show the histogram of the NW orientations computed from -90 to $90^{\circ}$ (Figure 1c). We find nine quantized orientations separated by 


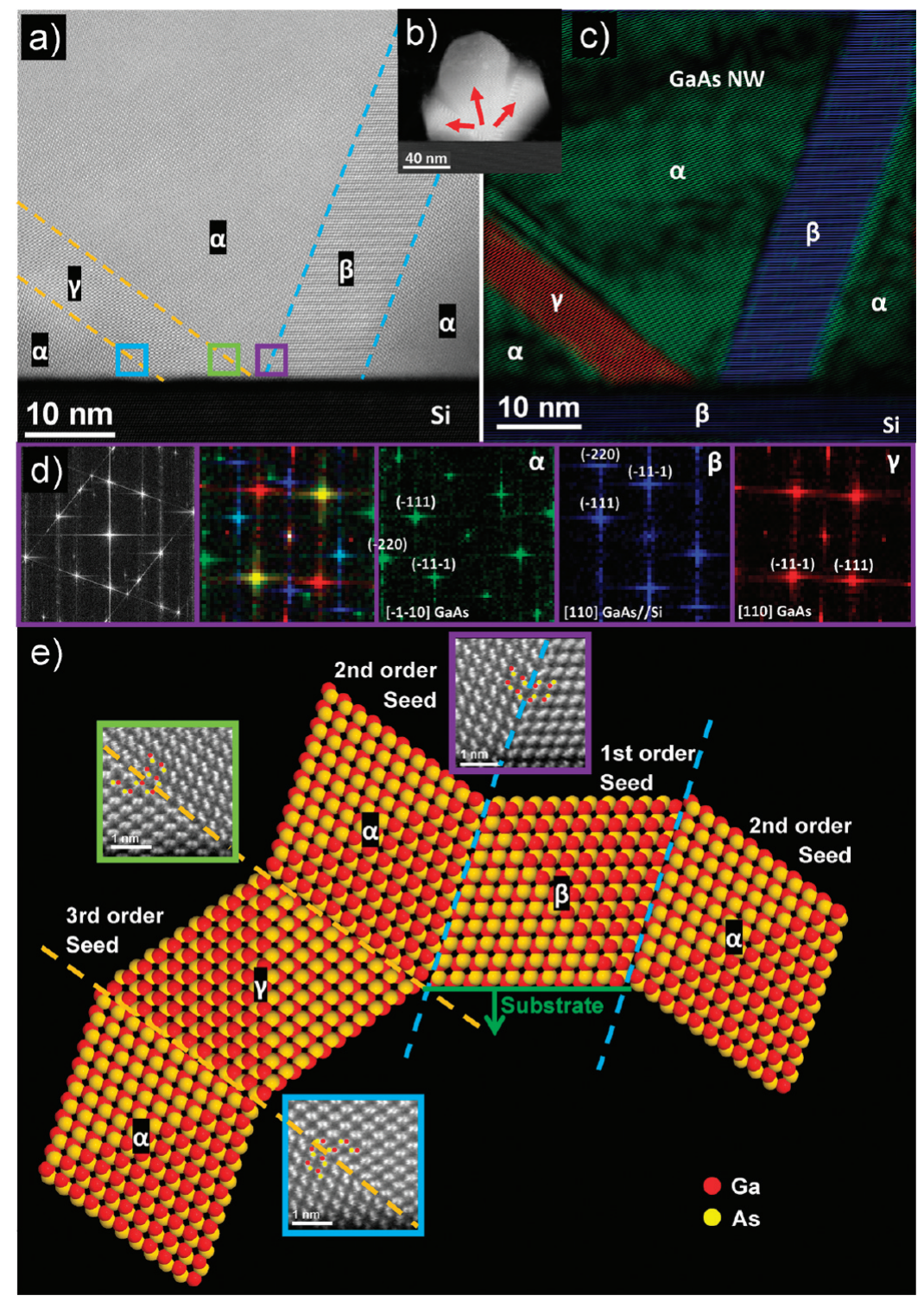

Figure 3. (a) Atomic HAADF STEM micrograph of the initial stages of GaAs nanowires exhibiting a multiple crystalline seed structure. (b) Low magnification TEM image of the sample, showing the three nanowire growth directions. (c) Visualization of the different crystal orientations by coloring them according to their characteristic frequency spots in the power spectra. For clarity, we show the filtered images. (d) Power spectra obtained in each of the crystalline regions of $\alpha, \beta, \gamma$. (e) Simulation of the atomic positions of the crystalline regions of $\alpha, \beta, \gamma$, showing the direction of the twinning and formation of secondary octahedral. The red and yellow spheres correspond to Ga and As atoms, respectively. The polarity analyses have been performed in detail in each of the twin boundaries (for more details see S2, Supporting Information). We find twins always perpendicular to the nanowire growth axis, and we never observe a change of the polarity (orthotwins).

about $\pm 19^{\circ}$ from the three main peaks, instead of the three expected originally. As will be shown in the following, these angles can only be explained with the formation of multiple seeds at the initial stages of the nanowire growth, a consequence of a 3D twinning phenomenon. Experimental evidence and theoretical details of this model are presented here below.

Before we proceed with the understanding of this phenomenon and for the sake of clarity, we draw schematically the occurrence of these two types of growth in Figure 2. The nanowires labeled as type I correspond to the ones for which there is a clear crystallographic relation between the growth direction and substrate. These are nanowires that grow perpendicularly with the substrate (seed nucleating with B polarity) and with a $19^{\circ}$ angle (seed nucleating with A polarity). For nanowires type II, such relation is not clear. As it will be shown in the following, for a clear understanding of the occurrence of these other angles, one needs to analyze in great detail the crystallography at the initial phases of growth.

To discover the underlying mechanism for the different growth angles, it has been key to analyze the crystalline phase of a group of nanowires growing from the same stem and branching toward distinctly different directions (Figure 2). STEMHAADF imaging was performed on a probe aberration corrected microscope (FEI Titan $60-300 \mathrm{kV}$ ), which allows us to analyze the elemental composition at the atomic level. A polarity analysis of the different branches of the NW seed, ${ }^{35}$ has to our knowledge not been reported before. In Figure panels a and b of 3, we show the existence of different grains in the seed, separated by twins at 

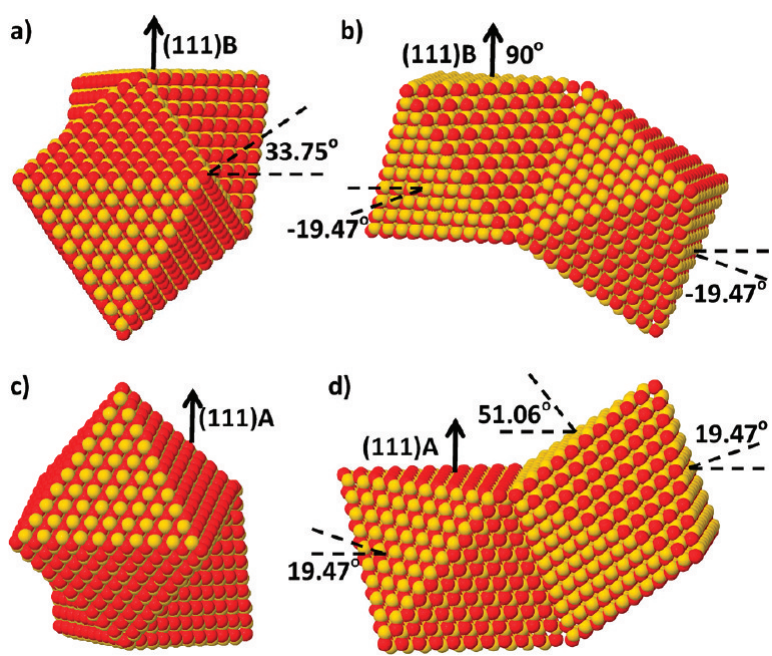

Figure 4. 3D atomistic models of the structures obtained when secondary crystalline seed forms from 3D twinning in one of the (111)B facets of an individual crystalline seed exhibiting either B (a, b) or A (c, d) polarity. The formation of secondary seeds opens up the possibility of other growth angles than the ones expected from a single crystalline seed $\left(90\right.$ and $\left.19.47^{\circ}\right)$, such as 33.75 and $51.06^{\circ}$.

$\{111\}$ planes. The corresponding interface exhibits 34 or $71^{\circ}$ with respect to the surface, giving rise to other grains, which we denote $\alpha, \beta$, or $\gamma$, depending on the orientation with respect to the substrate. The power spectra of these zones obtained by calculating the fast Fourier transform (FFT) are shown in the middle of the figure, indicating the orientations. For clarity, we have marked each of the grains with different colors by using frequency filtering in the FFT. Please note that neighboring grains share at least one common (111) plane. To indicate this, we use the color "green" when "yellow" and "blue" orientations are combined as marked in the respective FFTs. Given the threedimensional aspect of the seeds, it is not straightforward to obtain the spatial orientation of the planes from an image projection from HAADF-STEM. Note that STEM is a technique with a low focus depth, typically 5-10 nm. As will be shown in the following, three-dimensional modeling of the structure is necessary for the interpretation of the measurements. Grain $\beta$ exhibits a B polarity and it is the only to have an epitaxial relation with the Si substrate. The nanowire branches also grow following the B polarity, as expected from self-catalyzed GaAs nanowires. Surprisingly, although the [111]B growth direction is preserved, some of the NWs take other angles than what one would expect from a monocrystalline seed.

As we will see, twinning and further growth on the lateral facets results in the formation of secondary (multiple-order twinning) seeds that enable other [111]B crystal orientations. Its origin is related to the very first steps of the nanowire growth. The grains $\alpha$ on the left and right of Figure $3 \mathrm{a}-\mathrm{c}$ exhibit a $180^{\circ}$ rotation along the $[-111]$ direction with respect to $\beta$. This corresponds to the formation of a twin that conserves the polarity of the structure. From this twin a new crystal grain grows from the preferred [111]B direction; see Figure 1. Now, the $\{111\} \mathrm{B}$ type facets exhibit a different position in space than the original seed. The formation of the secondary grain allows the nanowires to grow in the directions of $34^{\circ}$ and $51^{\circ}$ (see Figures 2 and 4). A further twin in the grain $\alpha$ leads to the grain $\gamma$, separated by a twin that has an angle of $34^{\circ}$ with the substrate.
The possibilities of creating new surfaces are extremely large as they can increase by the creation of further seed crystals attached. One can predict the novel growth directions by obtaining the mirror image of the original seed along the twin plane and conserving the polarity of the crystal along the direction perpendicular to the twin. This operation is mathematically expressed by combining the Householder reflection matrices corresponding to the four (111) planes with an inversion operation with respect to the origin

$$
\begin{aligned}
& T_{(111)}=\left(\begin{array}{ccc}
-1 & 2 & 2 \\
2 & -1 & 2 \\
2 & 2 & -1
\end{array}\right), \\
& T_{(1 \overline{1} \overline{1})}=\left(\begin{array}{ccc}
-1 & -2 & -2 \\
-2 & -1 & 2 \\
-2 & 2 & -1
\end{array}\right), \\
& T_{(\overline{1} 1 \overline{1})}=\left(\begin{array}{ccc}
-1 & -2 & 2 \\
-2 & -1 & -2 \\
2 & -2 & -1
\end{array}\right), \\
& T_{(\overline{1} \overline{1} 1)}=\left(\begin{array}{ccc}
-1 & 2 & -2 \\
2 & -1 & -2 \\
-2 & -2 & -1
\end{array}\right)
\end{aligned}
$$

The growth orientations resulting from $n$-fold $3 \mathrm{D}$ twinning are obtained by multiplication of $n$ times of all combinations of the matrices $T_{j}$ with the $[111] \mathrm{B}$ directions, $d_{i}$, of the initial seed:

$$
t_{i, j}=T_{j} d_{i}
$$

Seeds nucleating with A and B polarity with respect to the silicon surface have to be considered separately, and we suppose that polarity is conserved upon twinning. The angles that define the resulting growth directions are: $\alpha$, the angle with respect to the surface and $\varphi$, the angle with the in plane direction $\langle 11-2\rangle$ when observed in planar view (from top). The calculated angles for all $\langle 111\rangle \mathrm{B}$ growth directions that are pointing out of the substrate surface are summarized in Table 1 up to the formation of a fourth order twinning phenomenon, for seeds with both a (111)B and (111)A polarity. We start by discussing the angles resulting from a single seed. For seeds nucleating with the (111)B polarity, vertical growth is obtained by following the direction of the nucleation seed. In this case, $\alpha$ is $90^{\circ}$ and there is no $\varphi$ defined. If now one considers the three lateral (111)B planes of the octahedral seed, we obtain three possible growth directions with $\alpha=-19.47^{\circ}$. Each one of these three lateral facets will exhibit one of the planar angles with $\varphi=60^{\circ}, 180^{\circ}$, and $300^{\circ}$. In contrast to this, considering a seed with a (111)A oriented octahedron, vertical growth is not possible. There are three lateral (111)B facets facing up at $19.47^{\circ}$ versus the horizontal from the substrate with $\varphi=0^{\circ}, 120^{\circ}$, and $240^{\circ}$, respectively. For higher order $3 \mathrm{D}$ twinning, the options for growth directions increase in a potential way.

By measuring $\alpha$ and/or $\varphi$, it is possible to identify the threedimensional twinning type. The origin of the growth angles different than $90,19.47$, or $-19.47^{\circ}$ (e.g., $34^{\circ}$ or $51^{\circ}$ ) is inconsistent with the crystallography of a single seed and can only be explained with a 3D twinning phenomenon. For example, the $\alpha=34$ 
Table 1. Orientation of the Nanowires Depending on the Nucleation Polarity and the Type of Facet Leading to Growth (top or lateral $)^{a}$

\begin{tabular}{|c|c|c|c|c|c|c|}
\hline (1) & $\begin{array}{l}\text { Seed } \\
\text { type }\end{array}$ & $\alpha$ & \multicolumn{4}{|c|}{$\varphi$ in-plane angles to $[11-2],[1-21],[-211]$} \\
\hline \multirow[t]{2}{*}{ 1st order } & A & 19.47 & 60.00 & & & \\
\hline & B & 90.00 & - & & & \\
\hline \multirow[t]{3}{*}{ 2nd order } & A & 19.47 & 0.00 & & & \\
\hline & B & 33.75 & 19.11 & -19.11 & & \\
\hline & A & 51.06 & 0.00 & & & \\
\hline \multirow[t]{6}{*}{ 3rd order } & A & 2.12 & 33.00 & -33.00 & 5.21 & -5.21 \\
\hline & B & 24.04 & 36.59 & -36.59 & & \\
\hline & $\mathrm{B}$ & 33.75 & 40.89 & -40.89 & & \\
\hline & A & 51.06 & 60.00 & & & \\
\hline & B & 58.41 & 60.00 & & & \\
\hline & A & 67.81 & 13.90 & -13.90 & & \\
\hline \multirow[t]{13}{*}{ 4th order } & A & 2.12 & 27.00 & -27.00 & -54.79 & 54.79 \\
\hline & $\mathrm{B}$ & 3.54 & 29.00 & -29.00 & 44.18 & -44.18 \\
\hline & B & 12.12 & 0.00 & 38.21 & -38.21 & \\
\hline & A & 13.57 & 8.95 & -8.95 & & \\
\hline & A & 22.50 & 40.89 & -40.89 & & \\
\hline & $B$ & 24.04 & 23.41 & -23.41 & & \\
\hline & $\mathrm{A}$ & 32.06 & 27.64 & -27.64 & 45.54 & -45.54 \\
\hline & $B$ & 40.87 & 28.68 & -28.68 & 53.11 & -53.11 \\
\hline & B & 53.37 & 54.18 & -54.18 & & \\
\hline & $\mathrm{A}$ & 55.81 & 15.61 & -15.61 & 53.82 & -53.82 \\
\hline & B & 58.41 & 0.00 & & & \\
\hline & A & 67.81 & 46.10 & -46.10 & & \\
\hline & B & 71.92 & 17.00 & -17.00 & & \\
\hline
\end{tabular}

${ }^{a} \alpha$ refers to the angle with the surface and $\varphi$ to the angle with the equivalent $\langle 11-2\rangle$ directions, which coincides with the projection of the [111] direction seen in a top view image. For a reference, a drawing of the angles on a substrate is shown.

and $51^{\circ}$ angles correspond to second order seeds created by the $3 \mathrm{D}$ twinning. Also, we can come back to the histogram in Figure $1 \mathrm{c}$. The three main peaks at 0 and $\pm 60^{\circ}$ correspond to nanowires growing in $\alpha= \pm 19.45^{\circ}$, from first generation seeds. The other quantized directions are mainly found at a distance $\Delta \varphi$ of $\pm 19^{\circ}$ from the main peaks. According to Table 1, these angles correspond to nanowires growing from second generation seeds, with $\alpha=33.75^{\circ}$. Although both the cross section and top view analysis are correct, the latter is much less subject to errors than cross-section measurements and allows for the realization of a statistical quantification in an easier way.

Finally, we discuss the occurrence of vertical, horizontal, and kinked nanowires as a function of the growth conditions. We find that the primary reason for the various nanowire orientations is related to the change in the size of the Ga droplet at the initial stage of the nanowire growth. We observe that by varying the $\mathrm{V} / \mathrm{III}$ ratio from 15 to 60 , with the Ga rate remaining the same, the incubation time varies from $2 \mathrm{~min}$ to $20 \mathrm{~s}$. This increase in the nucleation time must result in a larger Ga droplet, meaning that changes in the relative size of the Ga droplet at the initial stages of growth can be controlled by varying the $\mathrm{V} / \mathrm{III}$ ratio ${ }^{20}$ and temperature. It is especially important to control the size of the Ga droplet at the initial stages of growth where the seed(s) is(are) being formed. The Ga droplet increases rapidly in size, favoring the formation of new $\{111\}$ facets. In the case of a multiple-order twinned seed, the droplet can fall over to the side and be kinked. Even horizontal growth can occur. We find that lowering the $\mathrm{V} / \mathrm{III}$ ratio results in a broadening of the histograms due to the occurrence of third order twinning, while on increasing the $\mathrm{V} / \mathrm{III}$ ratio to 60 the second order twinning disappears (see S3, Supporting Information). To prohibit multiple-order twinning, it is therefore key to implement a high V/III ratio at least at the initial stage of growth and to keep the droplet size small enough. Very preliminary experiments indicate that $100 \%$ vertical wires are obtained when the $\mathrm{Ga}$ droplet is as small as $\sim 10 \mathrm{~nm}$.

In conclusion, we have found that polycrystalline seeds can occur at the initial stages of nanowire growth. The polycrystalline seed is formed by a three-dimensional twinning process. By taking into account the polarity, we deduce the geometrical rules leading to multiple-order 3D twinning, which are consistent with experimental results. These results are important for the reproducible integration of self-catalyzed III-V nanowires on group IV substrates. Moreover, this work opens the avenue for controlling the degree of complexity of nanowire networks. The understanding provided here gives a clear pathway of how to obtain fully vertical nanowire arrays, which would dramatically improve nanowire array devices such as solar or water splitting cells. Additionally, it shows how more complex branched nanowire structures could be grown, which can be useful for other 
applications such as interconnected nanoelectronic devices or cell cultures. ${ }^{36}$

\section{ASSOCIATED CONTENT}

S Supporting Information. Polarity of single seeds and consequences for the orientation of self-catalyzed nanowires, detailed analysis of the polarity of the nanowires and at the twin interface, and statistical analysis of the nanowire orientation as a function of the V/III ratio. This material is available free of charge via the Internet at http://pubs.acs.org

\section{AUTHOR INFORMATION}

\section{Corresponding Author}

*E-mail: anna.fontcuberta-morral@epfl.ch.

\section{Author Contributions}

TEqual contribution.

\section{ACKNOWLEDGMENT}

E.U., E.R.A., and A.F.iM. are grateful for funding through the ERC starting grant "Upcon" and the NCCR on "Quantum Science and Technology”. This work was partially supported by the Spanish Government projects Consolider Ingenio 2010 CSD2009 00013 IMAGINE and CSD2009 00050 MULTICAT. J.A. acknowledges the funding from the Spanish MICINN project MAT2010-15138 (COPEON) and Generalitat de Catalunya (2009 SGR 770 and XaRMAE). The authors would like to thank the TEM facilities in Laboratorio de Microscopias Avanzadas (LMA) of Instituto de Nanociencia de Aragon (INA) at Universidad de Zaragoza.

\section{REFERENCES}

(1) Bakkers, E. P. A. M.; Van Dam, J. A.; De Franceschi, S.; Kouwenhoven, L. P.; Kaiser, M.; Verheijen, M.; Wondergem, H.; van der Sluis, P. Nat. Mater. 2004, 3, 769-772.

(2) Martensson, T.; Patrik, C.; Svensson, T.; Wacaser, B. A.; Larsson, M. W.; Seifert, W.; Deppert, K.; Gustafsson, A.; Wallenberg, L. R.; Samuelson, L. Nano Lett. 2004, 4, 1987-1990.

(3) Chen, R.; Tran, T. T. D.; Ng, K. W.; Ko, W. S.; Chuang, L. C.; Sedwick, F. G.; Chang-hasnain, C. Nat. Photonics 2011, 5, 170-175.

(4) Chuang, L. C.; Moewe, M.; Chase, C.; Kobayashi, N. P.; Chang-Hasnain, C.; Crankshaw, S. Appl. Phys. Lett. 2007, 90, 043115.

(5) Krogstrup, P.; Popovitz-Biro, R.; Johnson, E.; Madsen, M. H.; Nygård, J.; Shtrikman, H. Nano Lett. 2010, 10, 4475-4482.

(6) Bjork, M. T.; Schmid, H.; Bessire, C. D.; Moselund, K. E.; Ghoneim, H.; Karg, S.; Lortscher, E.; Riel, H. Appl. Phys. Lett. 2010, $97,163501$.

(7) Glas, F. Phys. Rev. B 2006, 74, 121302.

(8) Mandl, B.; Stangl, J.; Mårtensson, T.; Mikkelsen, A.; Eriksson, J.; Karlsson, L. S.; Bauer, G.; Samuelson, L.; Seifert, W. Nano Lett. 2006, 6, 1817-1821.

(9) Wei, W.; Bao, X. Y.; Soci, C.; Ding., Y.; Wang, Z. L.; Wang, D. Nano Lett. 2009, 9, 2926.

(10) Cirlin, G. E.; Dubrovskii, V. G.; Soshnikov, I. P.; Sibirev, N. V.; Sammsonenko, Y. B.; Bouravleuv, A. D.; Harmand, J. C.; Glas, F. Phys. Status Solidi RRL 2009, 3, 112-114.

(11) Huang, H.; Ren, X.; Ye, X.; Guo, J.; Wang, Q.; Yang, Y.; Cai, S.; Huang, Y. Nano Lett. 2010, 10, 64-68.

(12) Tomioka, K.; Motohisa, J.; Hara, S.; Hiruma, K.; Fukui, T. Nano Lett. 2010, 10, 1639-1644.
(13) Boettcher, S. W.; Spurgeon, J. M.; Putnam, M. C.; Warren, E. L.; Turner-Evans, D. B.; Kelzenberg, M. D.; Maiolo, J. R.; Atwater, H. A.; Lewis, N. S. Science 2010, 327, 185-187.

(14) Kelzenberg, M. D.; Boettcher, S. W.; Petykiewicz, J. A.; Turner-Evans, D. B.; Putnam, M. C.; Warren, E. L.; Spurgeon, J. M.; Briggs, R. M.; Lewis, N. S.; Atwater, H. A. Nat. Mater. 2010, 9, 239.

(15) Czaban, J. A.; Thompson, D. A.; Lapierre, R. R. Nano Lett. 2009, 9, 148-154.

(16) Glas, F.; Harmand, J. C.; Patriarche, G. Phys. Rev. Lett. 2007, $99,146101$.

(17) Wen, C. Y.; Tersoff, J.; Reuter, M. C.; Stach, E. A.; Ross, F. M. Phys. Rev. Lett. 2010, 105, 19502.

(18) Glas, F.; Harmand, J.-C.; Patriarche, G. Phys. Rev. Lett. 2010, 104, 135501.

(19) Plissard, S.; Larrieu, G.; Wallart, X.; Caroff, P. Nanotechnology 2011, 22, 275602.

(20) Krogstrup, P.; Curiotto, S.; Johnson, E.; Aagesen, M.; Nygard, J.; Chatain, D. Phys. Rev. Lett. 2011, 106, 125505.

(21) Schwarz, K. W.; Tersoff, J. Nano Lett. 2011, 11, 316.

(22) Johansson, J.; et al. Nat. Mater. 2006, 5, 574-580.

(23) Korgel, B. A. Nat. Mater. 2006, 5, 521.

(24) Davidson, F. M.; Lee, D. C.; Dayne, D.; Fanfair, D. D.; Korgel, B. A. J. Phys. Chem. C 2007, 111, 2929-2935.

(25) Bao, J. M.; Bell, D. C.; Capasso, F.; Wagner, J. B.; Martensson, T.; Tragardh, J.; Samuelson, L. Nano Lett. 2008, 8, 836-841.

(26) Spirkoska, D.; Arbiol, J.; Gustafsson, A.; Conesa-Boj, S.; Zardo, I.; Heigoldt, M.; Gass, M. H.; Bleloch, A. L.; Estrade, S.; Peiro, F.; Morante, J. R.; Abstreiter, G.; Samuelson, L.; Fontcuberta i Morral, A. Phys. Rev. B 2009, 80, 245325.

(27) Thelander, C.; Caroff, P.; Plissard, S.; Dey, A. W.; Dick, K. A. Nano Lett. 2011, 11, 2424-2429.

(28) In the case where the polarity is conserved, the twin is known as "orthotwin". When it is not conserved, one speaks of "paratwin"; see ref 29 for all the details.

(29) Cohen, D.; McKernan, S.; Carter, C. B. Microsc. Microanal. 1999, 5, 173-186.

(30) Bakkers, E. P. A. M.; Van Dam, J. A.; De Franceschi, S.; Kouwenhoven, L. P.; Kaiser, M.; Verheijen, M.; Wondergem, H.; van der Sluis, P. Nat. Mater. 2004, 3, 769-772.

(31) Mårtensson, T.; Patrik, C.; Svensson, T.; Wacasar, B. A.; Larsson, M. W.; Seifert, W.; Deppert, K.; Gustafsson, A.; Wallenberg, L. R; Samuelson, L. Nano Lett. 2004, 4, 1987-1990.

(32) Colombo, C.; Spirkoska, D.; Frimmer, M.; Abstreiter, G.; Fontcuberta i Morral, A. Phys. Rev. B 2008, 77, 155326.

(33) Fontcuberta i Morral, A.; Colombo, C.; Abstreiter, G.; Arbiol, J.; Morante, J. R. Appl. Phys. Lett. 2008, 92, 063112.

(34) Bernal, S.; Botana, F. J.; Calvino, J.J.; López Cartes, C.; Pérez Omil, J. A.; Rodríguez-Izquierdo, J. M. Ultramicroscopy 1998, 72, 135.

(35) Robb, P. D.; Craven, A. J. Ultramicroscopy 2008, 109, 61-69.

(36) Rauber, M.; Alber, I.; Müller, S.; Neumann, R.; Picht, O.; Roth, C.; Schükel, A.; Toimil-Molares, M. E.; Ensinger, W. Nano Lett. 2011, 11, 2304-2310. 